\title{
Cold Storage Exacerbates Renal and Mitochondrial Dysfunction Following Transplantation
}

\author{
Shrum S, MacMillan-Crow LA and Parajuli N*
}

Department of Pharmacology and Toxicology, University of Arkansas for Medical Sciences, Little Rock, AR, USA

\begin{abstract}
Long-term renal function is compromised in patients receiving deceased donor kidneys which require cold storage exposure prior to transplantation. It is well established that extended cold storage induces renal damage and several labs, including our own, have demonstrated renal mitochondrial damage after cold storage alone. However, to our knowledge, few studies have assessed renal and mitochondrial function after transplantation of rat kidneys exposed to short-term (4 hr) cold storage compared to transplant without cold storage (autotransplantation). Our data reveal that cold storage plus transplantation exacerbated renal and mitochondrial dysfunction when compared to autotransplantation alone.
\end{abstract}

Keywords: Kidney; Mitochondria; Cold storage; Transplantation

\section{Introduction}

Kidney transplantation is the preferred modality of treatment for end stage kidney disease. While advances in tissue-type matching and immunosuppressive protocols have greatly reduced short-term graft dysfunction after renal transplantation, long-term graft function continues to be problematic, especially in patients receiving deceased donor kidneys [1-3]. The kidneys of deceased donors are routinely flushed and stored in cold storage (CS) solutions [4]. Short-term CS lowers the metabolic demand, yet, prolonged CS causes extensive tissue damage and is associated with a higher incidence of delayed graft function and poor long-term outcome after transplant $[1,5,6]$. Despite this, the mechanism of how CS negatively impacts overall graft function and survival is not well understood. Many laboratories, including our own, have published that renal CS (in vitro and ex vivo) induces mitochondrial injury, reactive oxygen species (ROS) generation, and cell death [7-12]. However, characterization of mitochondrial function after renal CS plus transplantation remains unknown. The respiratory enzyme complexes (complexes I-V) comprise the electron transfer chain, and through mitochondrial respiration generate ATP as the terminal product with ROS as a byproduct. When mitochondrial dysfunction occurs, cells are deprived of ATP and ROS are increased, consequently impacting numerous cellular pathways responsible for tissue regeneration/repair or damage. Therefore, our goal was to evaluate the contribution of short-term CS to early renal dysfunction and altered mitochondrial respiratory function following CS plus transplantation (CS/Tx) by comparing to autotransplant (ATx) (without CS exposure), and sham surgery.

\section{Methods}

\section{Animals}

Male Lewis rats weighing 200 - 250 g were used as transplant donors and recipients in this study. All of the animal protocols were approved by the Institutional Animal Care and Use Committee (IACUC) at the University of Arkansas for Medical Sciences (UAMS), and all animal experiments described below were performed in compliance with the IACUC at UAMS using NIH guidelines.

\section{Syngeneic rat renal transplant model with cold storage}

Orthotopic renal transplant surgery was performed in male Lewis rats. For the donor rat surgery, rats were anesthetized using isoflourane, and the left kidney was flushed with cold saline solution containing heparin (100 units/ml) via the aorta. The left kidney was removed en bloc (with the renal artery, vein and ureter attached) and stored in University of Wisconsin (UW) solution at $4^{\circ} \mathrm{C}$ for $4 \mathrm{hr}$. For the recipient rat surgery, rats were anesthetized using isoflourane, the native left kidney was removed, and the donor left kidney was transplanted using end-to-end anastomosis as described previously. The surgical ischemia time was $\sim 45 \mathrm{~min}$. The right native kidney was immediately removed, making renal function dependent on the transplanted left kidney. The ureter was anastomosed end-to-end over a $5 \mathrm{~mm}$ PE-50 polyethylene stent. Postoperatively, the animals were given $0.9 \%(\mathrm{w} / \mathrm{v}) \mathrm{NaCl}$ in the abdominal cavity and placed under a heating lamp to recover from the anesthesia. Rats were given buprenorphine $(0.06 \mathrm{mg} / \mathrm{kg}, \mathrm{SC})$ for pain. After $24 \mathrm{hr}$ of reperfusion, the transplanted left kidney and blood were collected under anesthesia and saved as $\mathbf{4 h r}$ cold storage plus transplantation (4h CS/Tx) group $(\mathrm{n}=4)$.

\section{Control groups}

Autotransplant surgery: The autotransplant (ATx) surgery was performed as described in the recipient surgery method, except that the left kidney was removed and immediately transplanted in the same rat without CS exposure, followed by right nephrectomy. The surgical ischemia time was $\sim 45 \mathrm{~min}$. After $24 \mathrm{hr}$, the transplanted kidney was harvested under anesthesia, and the organs were referred to as Autotransplantation (ATx) group $(\mathrm{n}=4)$.

Sham surgery: Rats underwent identical surgery (right nephrectomy), but without the renal transplantation (Sham operation). The right kidney from a donor rat was removed and the left kidney remained functioning for $24 \mathrm{hr}$, and then the sham kidney and blood were harvested and saved as the Sham group $(n=4)$.

*Corresponding author: Nirmala Parajuli, Ph.D., University of Arkansas for Medical Sciences, 325 Jack Stephens Drive, Biomedical Bldg. I 333, Little Rock, AR 72205, USA, Tel: 501-686-5290; Fax: 501-686-8970; E-mail: nparajuli@uams.edu

Received: December 04, 2015; Accepted: January 27, 2016; Published: February 27, 2016

Citation: Shrum S, MacMillan-Crow LA, Parajuli N (2016) Cold Storage Exacerbates Renal and Mitochondrial Dysfunction Following Transplantation. J Kidney 2: 114. doi:10.4172/2472-1220.1000114

Copyright: @ 2016 Shrum S, et al. This is an open-access article distributed unde the terms of the Creative Commons Attribution License, which permits unrestricted use, distribution, and reproduction in any medium, provided the original author and source are credited. 


\section{Blood chemistry}

Blood chemistry was determined in heparinized blood (arterial) using a hand-held clinical chemistry analyzer, iSTAT ${ }^{\mathrm{Tx}}$ [13], and cartridges $\left(\mathrm{CHEM}^{+}\right)$as described by the manufacturer (Vetscan;, Abaxis, USA).

\section{High resolution respirometry (HRR)}

Mitochondrial respiratory complex activity was measured in the saponin permeabilized renal biopsies by high resolution respirometry (HRR) (Oroboros instruments - Oxygraph-2k, Innsbruck, Austria), according to substrate-inhibitor-titration (SIT) protocol as described earlier [11,14]. DATLAB 4.2 software (Oroboros) was used to analyze data, and tissue respiration was shown as oxygen flux ( $\mathrm{pmol} / \mathrm{mg} / \mathrm{s})$.

\section{ATP assay}

An ATP-luciferase-based bioluminescence assay kit (Sigma, MO, USA) and TD 20/20 luminometer (Turner Designs Sunnyvale, CA, USA) were used to measure ATP levels in the sham and transplant kidney tissue lysates as described earlier [14].

\section{Statistical analysis}

Results are presented as mean \pm standard error of the mean (S.E.M.) using Graph Pad Prism software (version 4.0). The Student's $t$-test was used to compare differences between the mean of two groups at a $95 \%$ level of confidence. Differences with a $P$ value $<0.05$ were considered statistically significant. Comparisons were made between the groups: sham vs. ATx, sham vs. $4 \mathrm{hrCS} / \mathrm{Tx}$, and AutoTx vs. $4 \mathrm{hrCS} / \mathrm{Tx}$.

\section{Results and Discussion}

We previously reported in isolated rat and pig kidneys that CS alone induced significant renal and mitochondrial injury [10,11]. In this study, we evaluated renal function after $4 \mathrm{hr}$ CS combined with transplantation using a syngeneic transplantation model to minimize confounding effects of the host immune system. Similarly, the autotransplant model without CS was chosen to evaluate the effects of warm ischemia combined with surgical trauma on kidney and mitochondrial function following transplantation. Sham rats showed no significant changes in plasma creatinine $(\mathrm{Cr} ; \sim 0.5 \mathrm{mg} / \mathrm{dl})$ and blood urea nitrogen (BUN; $\sim 20 \mathrm{mg} / \mathrm{dl}$ ), but these parameters were increased after autotransplant (ATx; Figure 1A and 1B). However, rat kidneys exposed to $4 \mathrm{hr}$ CS plus transplantation showed markedly increased levels of $\mathrm{Cr}$ and BUN (4hrCS/Tx, Figure 1A and 1B).

Our laboratory previously showed that CS (4 hr) alone induces altered renal mitochondrial function (reduced respiratory complex I-IV activity, using a spectrophotometric assay and isolated renal mitochondria), leading to renal tubular injury [10]. However, it was not clear if mitochondrial damage extended to post-transplant. In the current study, High Resolution Respirometry (HRR) was used, which, in contrast to the spectrophotometric assay, monitors real-time mitochondrial respiration in fresh renal biopsies, thereby excluding the possibility of introducing assay artifacts on functional analysis via mitochondrial isolation.

A significant decline in renal mitochondrial complex I respiratory function $(\sim 50 \%)$ was detected by reduced oxygen flux following CS/Tx when compared to sham or ATx groups (Figure 2A). Similarly, complex III activities was significantly declined after CS/Tx when compared to ATx (Figure 2A). There was no change in complex II or IV activity
A.

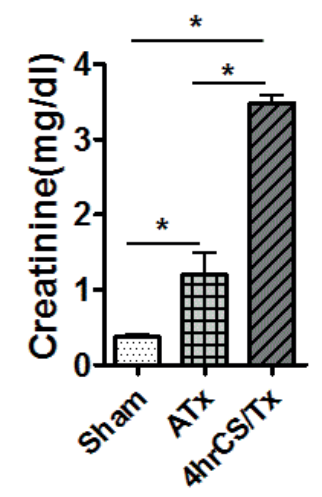

B.

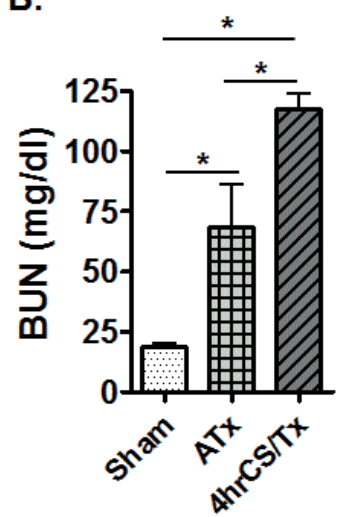

Figure 1: Cold storage worsens renal function after transplantation. Whole blood (arterial) creatinine and blood urea nitrogen (BUN) from the sham, autotransplant (ATx), and $4 \mathrm{hCS} / \mathrm{Tx}$ rats were analyzed using hand-held clinical chemistry analyzer (iSTAT ${ }^{\mathrm{TM}}$ ) and $\mathrm{Chem}^{+}{ }^{+}$cassettes as described in materials and methods. Values were expressed as Mean \pm S.E.M. $(n=3-4)$; ${ }^{*}$ indicates means are significantly different $(P<0.05)$.

\section{A. Renal Mitochondrial Respiration}

Complex I Complex II Complex III Complex IV

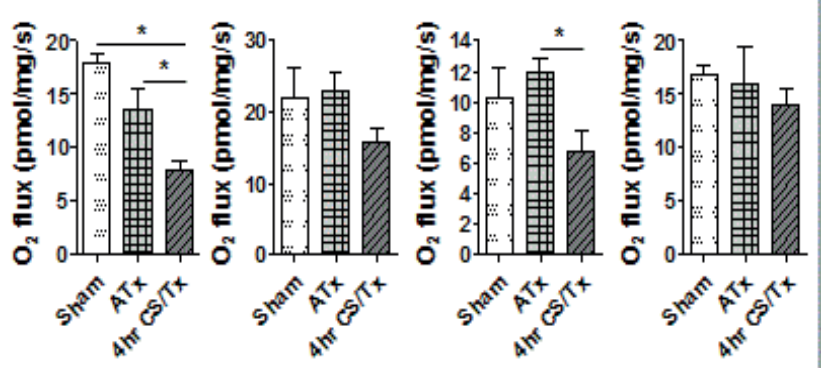

\section{B. Renal ATP level}

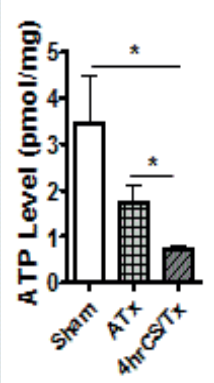

Figure 2: Cold storage worsens mitochondrial function after transplantation. (A) Graph showing respiratory complex I, II, III, and IV activity of the electron transport chain using high resolution respirometry in sham kidneys, or after transplantation with $4 \mathrm{hr}$ cold storage (CS/Tx) or without CS (autotransplant, ATx). (B) Graph showing ATP levels in renal homogenates in sham, CS plus transplantation (CS/ Tx), or autotransplant (ATx) groups. Error bar indicates Mean \pm S.E.M. $(n=3-4)$; *indicates means are significantly different $(P<0.05)$.

following CS/Tx when compared to sham or ATx. To our knowledge, this is the first report demonstrating a reduction of renal mitochondrial respiratory complex (I and III) activity following renal CS/Tx. Since compromised respiratory complex activity can lead to reduced ATP levels, we also evaluated ATP levels. CS plus transplantation showed 
Citation: Shrum S, MacMillan-Crow LA, Parajuli N (2016) Cold Storage Exacerbates Renal and Mitochondrial Dysfunction Following Transplantation. J Kidney 2: 114. doi:10.4172/2472-1220.1000114

a dramatic loss of renal ATP when compared to the sham or ATx ATP levels (Figure 2B). These data suggest that short-term (4 hr) CS negatively impacts mitochondrial function following transplantation.

Several other studies have documented renal dysfunction following various times of CS, although many are much longer than $4 \mathrm{hr}[4,15,16]$. Our study revealed, for the first time, that CS (4 hr) leads to a selective respiratory complex inhibition (I and III, but not II and IV) and ATP depletion following transplantation. Therefore, we anticipate that loss of mitochondrial function is a critical event that leads to energy depletion and exacerbated renal dysfunction during CS/Tx.

The profound decline in complex I activity occurring after CS/Tx is significant because complex I has been shown to be a major source of ROS in many diseases [17-19]. In addition, complex III, which was also inactivated after CS/Tx, also participates in ROS generation [2022]. Future studies will evaluate CS-induced oxidant generation and its involvement in the mechanism of respiratory complex inactivation following CS/Tx. Similarly, additional studies employing longer CS and reperfusion times are warranted to evaluate how CS negatively impacts mitochondrial function that causes sustained loss of ATP following $\mathrm{CS} / \mathrm{Tx}$. One limitation of the current study is that rat kidneys were harvested from living animals, whereas clinically the majority of kidney donors are harvested from deceased patients. Future studies could be performed using a non-heart beating donor rat prior to CS, but the rationale for the current study was to dissect the effect that CS alone has on mitochondrial damage. Another limitation is certainly the time of CS as well as reperfusion, and new studies are underway to determine the extent of mitochondrial/renal damage with longer CS times (up to $24 \mathrm{hr}$ ). Finally, new studies will need to look at 3-7 days post-transplant to determine if function is improved or worsened.

\section{Acknowledgement}

We thank National Institutes of Health $(\mathrm{NIH})$ and for financial support. We also would like to thank Pragya Singh for technical help.

\section{Grant}

National Institutes of Health (NIH) grants: RO1DK078936 (LAMC), T32DK061921 (NP), and T32 GM106999 (SS).

\section{References}

1. Debout A, Foucher Y, Trébern-Launay K, Legendre C, Kreis H, et al. (2015) Each additional hour of cold ischemia time significantly increases the risk of graft failure and mortality following renal transplantation. Kidney Int 87: 343349 .

2. Floerchinger B, Oberhuber R and Tullius SG (2012) Effects of brain death on organ quality and transplant outcome. Transplant Rev (Orlando) 26: 54-59.

3. Salahudeen AK (2004) Consequences of cold ischemic injury of kidneys in clinical transplantation. J Investig Med 52: 296-298.

4. Hoeger S, Lueg G, Tsagogiorgas C, Schneider M, Theisinger S, et al. (2011) UW is superior compared with HTK after prolonged preservation of renal grafts. J Surg Res 170: e149-157.

5. Hoeger S, Petrov K, Reisenbuechler A, Fontana J, Selhorst J, et al. (2009) The additional detrimental effects of cold preservation on transplantation-associated injury in kidneys from living and brain-dead donor rats. Transplantation 87: 52-58.
6. van der Vliet JA, Warlé MC (2013) The need to reduce cold ischemia time in kidney transplantation. Curr Opin Organ Transplant 18: 174-178.

7. Salahudeen AK, Huang H, Joshi M, Moore NA, Jenkins JK (2003) Involvement of the mitochondrial pathway in cold storage and rewarming-associated apoptosis of human renal proximal tubular cells. Am J Transplant 3: 273-280.

8. Green CJ, Healing G, Lunec J, Fuller BJ, Simpkin S (1986) Evidence of freeradical-induced damage in rabbit kidneys after simple hypothermic preservation and autotransplantation. Transplantation 41: 161-165.

9. Mitchell T, Saba H, Laakman J, Parajuli N, Macmillan-Crow LA (2010) Role of mitochondrial-derived oxidants in renal tubular cell cold-storage injury. Free Radic Biol Med 49: 1273-1282.

10. Mitchell T, Rotaru D, Saba H, Smith RA, Murphy MP, et al. (2011) The mitochondria-targeted antioxidant mitoquinone protects against cold storage injury of renal tubular cells and rat kidneys. J Pharmacol Exp Ther 336: 682692.

11. Parajuli N, Campbell LH, Marine A, Brockbank KG, Macmillan-Crow LA (2012) MitoQ blunts mitochondrial and renal damage during cold preservation of porcine kidneys. PLoS One 7: e48590.

12. Cekauskas A, Bruns H, Manikas M, Herr I, Gross ML, et al. (2013) Sulforaphane decreases kidney injury after transplantation in rats: role of mitochondrial damage. Ann Transplant 18: 488-496.

13. Mock T, Morrison D, Yatscoff R (1995) Evaluation of the i-STAT system: a portable chemistry analyzer for the measurement of sodium, potassium, chloride, urea, glucose, and hematocrit. Clin Biochem 28: 187-192.

14. Patil NK, Parajuli N, Macmillan-Crow LA, Mayeux PR (2014) Inactivation of renal mitochondrial respiratory complexes and manganese superoxide dismutase during sepsis: mitochondria-targeted antioxidant mitigates injury Am J Physiol Renal Physiol 306: F734-F743.

15. Goujon JM, Vandewalle A, Baumert H, Carretier M, Hauet T (2000) Influence of cold-storage conditions on renal function of autotransplanted large pig kidneys. Kidney Int 58: 838-850.

16. Sener A, Tran KC, Deng JP, Garcia B, Lan Z, et al. (2013) Carbon monoxide releasing molecules inhibit cell death resulting from renal transplantation related stress. J Urol 190: 772-778.

17. Garcia-Ruiz I, Fernandez-Moreira D, Solis-Munoz P, Rodriguez-Juan C, DiazSanjuan T, et al. (2010) Mitochondrial complex I subunits are decreased in murine nonalcoholic fatty liver disease: implication of peroxynitrite. J Proteome Res 9: 2450-2459.

18. He Y, Leung KW, Zhang YH, Duan S, Zhong XF, et al. (2008) Mitochondria complex I defect induces ROS release and degeneration in trabecular meshwork cells of POAG patients: protection by antioxidants. Invest Ophthalmol Vis Sci 49: $1447-1458$.

19. Kushnareva Y, Murphy AN, Andreyev A (2002) Complex I-mediated reactive oxygen species generation: modulation by cytochrome $c$ and $N A D(P)+$ oxidation-reduction state. Biochem J 368: 545-553.

20. Chandel NS, McClintock DS, Feliciano CE, Wood TM, Melendez JA, et al. (2000) Reactive oxygen species generated at mitochondrial complex III stabilize hypoxia-inducible factor-1alpha during hypoxia: a mechanism of $\mathrm{O} 2$ sensing. J Biol Chem 275: 25130-25138.

21. Chen Q, Vazquez EJ, Moghaddas S, Hoppel CL, Lesnefsky EJ (2003) Production of reactive oxygen species by mitochondria: central role of complex III. J Biol Chem 278: 36027-36031.

22. Muller FL, Liu Y, Van Remmen H (2004) Complex III releases superoxide to both sides of the inner mitochondrial membrane. J Biol Chem 279: 4906449073. 